J. Korean Math. Soc. 51 (2014), No. 6, pp. 1177-1187

http://dx.doi.org/10.4134/JKMS.2014.51.6.1177

\title{
ABSOLUTELY PURE REPRESENTATIONS OF QUIVERS
}

\author{
Mansour Aghasi and Hamidreza Nemati
}

\begin{abstract}
In the current paper we study absolutely pure representations of quivers. Then over some nice quivers including linear quivers some sufficient conditions guaranteeing a representation to be absolutely pure is characterized. Furthermore some relations between flatness and absolute purity is investigated. Finally it is shown that the absolutely pure covering of representations of linear quivers (including $\mathbb{A}_{\infty}^{-}, \mathbb{A}_{\infty}^{+}$and $\left.\mathbb{A}_{\infty}^{\infty}\right)$ by $R$-modules whenever $R$ is a coherent ring exists.
\end{abstract}

\section{Introduction}

The study of quivers and their representations by modules is a topic in temporary research in module and ring theory. In homological point of view it is important to consider some canonical classes of objects providing tools to compute cohomology. So far the class of projective representations of quivers in [3], of injective representations of quivers in [5], of flat representations of quivers in [6], of projective and injective representations of quivers with $n$ edges in [11], and of pure injective representations in [8] have been widely studied.

Absolutely pure $R$-modules were first considered by Admas in [1] and by Maddox in [9]. Megibben in [10] and Stenström in [13] and many other authors have continued the study of absolutely pure $R$-modules. Pinzon in [12] proved the existence of absolutely pure covering of $R$-modules. This paper is scheduled to study absolute purity in the category of representations of quivers. We first consider a kind of purity in the category of representations introduced and studied in [8]. Then by an absolutely pure representation we mean a representation $\mathcal{X}$ such that every exact sequence

$$
0 \rightarrow \mathcal{X} \rightarrow \mathcal{Y} \rightarrow \mathcal{Z} \rightarrow 0
$$

is pure. Notice that based on the argument in the introduction in [8], it is possible that the standard notion of purity in locally finitely presented categories and the kind of purity in [8] are two different notions. However it is not hard to show that the categorical purity is stronger than the other notions.

Received December 16, 2013; Revised May 31, 2014.

2010 Mathematics Subject Classification. 16G20, 16D90.

Key words and phrases. representations of a quiver, pure monomorphism, absolutely pure representations, flat representations. 
Over a quiver with finite number of vertices, these two notions of purity are equivalent. A representation $\mathcal{X}$ is called $\mathrm{Fp}$-injective if every monomorphism $0 \rightarrow \mathcal{X} \rightarrow \mathcal{Y}$ of representations is a pure monomorphism. In general the class of Fp-injective representations possibly differs from the class of absolutely pure representations.

We prove that the class of absolutely pure representations in $\operatorname{Rep}(Q, R)$, i.e., $\operatorname{Abs}(Q, R)$, has some nice properties such as being closed under direct sum, direct limits and pure subrepresentations. Then we use these to show that $\operatorname{Abs}(Q, R)$ where $R$ is a coherent ring is a filtration class and hence is a covering class.

\section{Preliminaries}

A quiver $Q$ is simply a directed graph. More precisely a quiver $Q$ is a fourtuple $Q=(V, E, s, t)$ where $V$ is the set of vertices, $E$ is the set of arrows, and $s, t: E \rightarrow V$ are two maps where for each arrow $a \in E, s(a)$ and $t(a)$ assign to $a$, the initial and terminal vertex of $a$ respectively.

A representation $\mathcal{X}$ by $R$-modules of a given quiver $Q$ is a functor $\mathcal{X}: Q \rightarrow$ $R-M$ od where $Q$ is considered as an small category whose objects are vertices of $Q$ and paths are its homomorphisms. $\mathcal{X}$ is determined by assigning a module $\mathcal{X}(v)$ to any vertex $v \in V$ and a homomorphism $\mathcal{X}(a): \mathcal{X}(v) \rightarrow \mathcal{X}(w)$ to each arrow $a: v \rightarrow w$. A morphism $\mathcal{X} \rightarrow \mathcal{Y}$ is a natural transformation. A representation $\mathcal{P}$ of $Q$ is said to be finitely presented if the functor $\operatorname{Hom}_{Q}(\mathcal{P},-)$ preserves direct limits. The representations of a quiver $Q$ by $R$-modules, denoted by $\operatorname{Rep}(Q, R)$ is a Grothendieck category with enough projectives.

A quiver $Q$ is called right(left) rooted if there is no path of the form $\bullet \rightarrow$ $\bullet \rightarrow \cdots(\cdots \rightarrow \bullet \rightarrow \bullet)$.

For any quiver $Q=(V, E)$, by $Q^{o p}=\left(V, E^{o p}\right)$ we mean a quiver with the same set of vertices and reversed arrows, i.e., if $a: v \rightarrow w$ is an arrow in $Q$, then $a^{o p}: w \rightarrow v$ is the corresponding arrow in $Q^{o p}$. Notice that a quiver $Q$ is left rooted if and only if $Q^{o p}$ is right rooted. For a given representation $\mathcal{X}$ of a quiver $Q, \mathcal{X}^{+}$is a representation in $\operatorname{Rep}\left(Q^{o p}, R\right)$ such that $\mathcal{X}^{+}(v)=\operatorname{Hom}(\mathcal{X}(v), \mathbb{Q} / \mathbb{Z})$.

The following two theorems are chosen from [6] and [5] respectively.

Theorem 2.1. For a left rooted quiver $Q$, a representation $F$ is flat if and only if the following hold.

(i) $F(v)$ is a flat $R$-module for every vertex $v$ in $Q$,

(ii) For all vertices $v$ of $Q$, the homomorphism $g_{v}: \oplus_{t(a)=v} F(s(a)) \rightarrow F(v)$ induced by $F(s(a)) \rightarrow F(v)$ is a pure monomorphism.

Proof. See Theorem 3.7 in [6].

Theorem 2.2. Let $Q$ be a left rooted quiver. Then $F$ is a flat representation of $Q=(V, E)$ if and only if $F^{+}$is an injective representation of $Q^{o p}=\left(V, E^{o p}\right)$.

Proof. See Corollary 6.7 in [5]. 
A continuous chain $\left(\mathcal{M}_{\alpha} \mid \alpha \leq \sigma\right)$ of subrepresntations of a representation $\mathcal{M}$ whenever $\sigma$ is an infinite ordinal number is called a filtration for $\mathcal{M}$. In this case we have $\mathcal{M}_{0}=0$, for $\alpha \leq \beta, \mathcal{M}_{\alpha} \subseteq \mathcal{M}_{\beta}$, and $\mathcal{M}_{\beta}=\cup_{\alpha \leq \beta} \mathcal{M}_{\alpha}$ whenever $\beta$ is a limit ordinal and $\mathcal{M}_{\sigma}=\mathcal{M}$. For a class $C$ of representations such a filtration is said to be a $C$-filtration if for every $\alpha<\sigma$ the represntation $\mathcal{G}_{\alpha+1} / \mathcal{G}_{\alpha}$ is isomorphic to an element of $C$. Some applications of filtrations to relative homological algebra and an exact definition of filtrations are presented in $[2]$.

Let $\mathcal{F}$ be a class of representations. A morphism $\phi: F \rightarrow M$ of representations with $F \in \mathcal{F}$ is called an $\mathcal{F}$ - precover of $M$ if the sequence $\operatorname{Hom}\left(F^{\prime}, F\right) \rightarrow$ $\operatorname{Hom}\left(F^{\prime}, M\right)$ of abelian groups for any $F^{\prime} \in \mathcal{F}$ is an epimorphism. $\phi$ is called $\mathcal{F}$-cover if it has the extra property that $\phi f=\phi$ holds only when $f: F \rightarrow F$ is an authomorphism.

\section{C-absolutely pure representations}

The category of representations of a quiver by modules is a locally finitely presented category. So one can consider a canonical definition of purity in this category. Also a possibly different notion of purity is introduced in [8]. Finally another notion of purity is related to vertices.

An exact sequence $\varepsilon: 0 \rightarrow \mathcal{X} \rightarrow \mathcal{Y} \rightarrow \mathcal{Z} \rightarrow 0$ is called;

(1) categorically pure if

$$
0 \rightarrow \operatorname{Hom}(\mathcal{F}, \mathcal{X}) \rightarrow \operatorname{Hom}(\mathcal{F}, \mathcal{Y}) \rightarrow \operatorname{Hom}(\mathcal{F}, \mathcal{Z}) \rightarrow 0
$$

is exact for each finitely presented representation $\mathcal{F}$,

(2) pure if $0 \rightarrow \mathcal{Z}^{+} \rightarrow \mathcal{Y}^{+} \rightarrow \mathcal{X}^{+} \rightarrow 0$ splits,

(3) componentwise pure (or C-pure) if $0 \rightarrow \mathcal{X}(v) \rightarrow \mathcal{Y}(v) \rightarrow \mathcal{Z}(v) \rightarrow 0$ is a pure exact sequence of $R$-modules for each vertex $v \in V$.

The next proposition is a comparison between the categorical notion and other notions of purity.

Proposition 3.1. In $\operatorname{Rep}(Q, R)$, i.e., the category of representations of a quiver $Q$, categorically pure short exact sequences are pure exact, that is,

$$
\text { Pure }_{\text {cat }} \subseteq \text { Pure }_{+} \text {. }
$$

Proof. Let $\varepsilon: 0 \rightarrow \mathcal{X} \rightarrow \mathcal{Y} \rightarrow \mathcal{Z} \rightarrow 0$ be a pure categorical short exact sequence. Since $\operatorname{Rep}(Q, R)$ is locally finitely presented, one can consider $\mathcal{Z}$ as a direct limit of some finitely presented representations $\underline{l i m}_{i}$ and hence $\varepsilon$ can be written as $\lim _{\longrightarrow} \varepsilon_{i}$ for some split short exact sequences $\overrightarrow{\varepsilon_{i}}$. By [8, Proposition $2.5]$, each $\varepsilon_{i}$ is pure exact and any direct limit of pure exact sequences is pure exact.

Remark 3.2.

(1) Consider the quiver $\bullet \rightarrow \bullet$ and two representations $\mathcal{M}: M \oplus M \stackrel{\text { id }}{\rightarrow}$ $M \oplus M$ and $\mathcal{M}^{\prime}: M \stackrel{\iota}{\rightarrow} M \oplus M$ where $\iota$ is the canonical inclusion. 
Although $\beta=(\iota, i d)$ is a monomorphism of representations with $\iota$ and $i d$ pure monomorphisms, $\beta$ is not a pure monomorphism of representations. This shows that componentwise purity does not yield purity in $\operatorname{Rep}(Q, R)$.

(2) When $Q$ has a finite number of vertices the path $\operatorname{ring} R Q$ has an identity and $\operatorname{Rep}(R, Q)$ and $R Q$-modules are equivalent categories. In this case any pure exact sequence of representations is categorically pure.

Let $Q$ be any quiver and let $\mathcal{M}$ be a representation of $Q . \mathcal{M}$ is called componentwise absolutely pure if $\mathcal{M}(v)$ is an absolutely pure $R$-module for any $v \in V$. We denote the class of all componentwise absolutely pure representations by $\operatorname{Cabs}(Q)$. Componentwise flat representations, $\operatorname{Cflat}(Q)$, is considered in [7]. The concepts of $\operatorname{Cflat}(Q)$ and $\operatorname{Cabs}(Q)$ are not the categorical definitions of flatness and absolute purity but they are relevant definitions when we consider $\operatorname{Rep}(Q, R)$ as the category of presheaves on a topological space.

Here we state Lemma 4.4 from [7].

Lemma 3.3. Let $\aleph$ be an infinite cardinal such that $\aleph \geq \sup \{|R|,|V|,|E|\}$ and $\mathcal{M}$ be a representation of $Q$. Then for each $x \in M$, there exists a $C$-pure subrepresentation $P$ of $M$ such that $x \in P$ and $|P| \leq \aleph$.

Theorem 3.4. Let $R$ be a coherent ring. Then the class of all componentwise absolutely pure representations, i.e., $\operatorname{Cabs}(Q)$ is a covering class.

Proof. Let $M$ be a representation and $x \in M$. Then by Lemma 3.3 there exists a C-pure representation $P_{1} \subseteq M$ such that $\left|P_{1}\right| \leq \aleph$. Hence $P_{1}$ and $M / P_{1}$ belong to $\operatorname{Cabs}(Q)$. Taking any element $x_{1} \in M / P_{1}$ we can find a $\mathrm{C}$ pure subrepresentation $P_{2} / P_{1}$ containing $x_{1}$ belonging to $\operatorname{Cabs}(Q)$ such that $\left|P_{2} / P_{1}\right| \leq \aleph$. Both of $P_{1}$ and $P_{2} / P_{1}$ belong to $\operatorname{Cabs}(Q)$ and hence $P_{2}$ and $M / P_{2}$ belong to $\operatorname{Cabs}(Q)$. For an ordinal $\alpha$ and considering $M / P_{\alpha}$, one can use the same procedure to get a C-pure subrepresentation $P_{\alpha} \subseteq P_{\alpha+1} \subseteq M$ such that $\left|P_{\alpha+1} / P_{\alpha}\right| \leq \aleph$. For a limit ordinal $\beta$ set $P_{\beta}=\lim _{\alpha<\beta} P_{\alpha}$. Then $\left(P_{\alpha}: \alpha<\lambda\right)$ for a suitable cardinal number $\lambda$ is a $\mathrm{C}$-filtration for $M$. It is not hard to see that any representation $M$ with a C-filtration belongs to $\operatorname{Cabs}(Q)$. Hence $\operatorname{Cabs}(Q)$ is a covering class by [2, Theorem 5.5].

\section{Absolutely pure representations}

An $R$-module $M$ is called absolutely pure if every short exact sequence

$$
0 \rightarrow M \rightarrow N \rightarrow P \rightarrow 0
$$

is pure. Absolutely pure modules were first studied by Adams in [1] and developed by several authors such as Megibben in [10], Maddox in [9] and Stenström in [13], etc. Recently Pinzon in [12] has proved that the category of $R$-modules admits absolutely pure coverings. In this section we study absolutely pure representations of quivers. 
Definition. Let $Q$ be any quiver and $\operatorname{Rep}(Q, R)$ denotes the category of representations of $Q$ in $R$-Mod. A representation $\mathcal{X}$ is called absolutely pure if every exact sequence $0 \rightarrow \mathcal{X} \rightarrow \mathcal{Y}$ is pure. $\mathcal{X}$ is called componentwise absolutely pure if $\mathcal{X}(v)$ is an absolutely pure $R(v)$-module for each vertex $v \in Q$.

Example 1. It will be proved that any absolutely pure representation is componentwise absolutely pure. Let $Q: \bullet \rightarrow \bullet$ be a quiver and $M \neq 0$ be an absolutely pure $R$-module with injective envelope $E$. Then $0 \rightarrow M$ is componentwise absolutely pure. It is not difficult to show that $(0 \rightarrow M) \rightarrow(E \rightarrow E)$ is a monomorphism which is not pure. Hence $0 \rightarrow M$ is not absolutely pure. Representations $M \stackrel{i d}{\rightarrow} M$ and $0 \rightarrow M$ are examples of absolutely pure representations of $Q$.

By using Example 2.4 in [8] one can show that any componentwise absolutely pure representation $\mathcal{X}$ where $\mathcal{X}(v) \rightarrow \mathcal{X}(w)$ is a split epimorphism for each arrow $v \rightarrow w$ is absolutely pure.

Lemma 4.1. The class of all absolutely pure representations is closed under taking pure sub-object.

Proof. Suppose that $\mathcal{X}$ be a pure subrepresentation of an absolutely pure representation $\mathcal{Y}$ and we are given an inclusion $0 \rightarrow \mathcal{X} \rightarrow \mathcal{A}$. Let $\mathcal{P}$ be the push-out of $\mathcal{X} \rightarrow \mathcal{Y}$ and $\mathcal{X} \rightarrow \mathcal{A}$. Then since $\mathcal{X} \subseteq \mathcal{Y} \subseteq \mathcal{P}$ is pure, so is the composition $\mathcal{X} \subseteq \mathcal{A} \subseteq \mathcal{P}$. Hence $\mathcal{X} \subseteq \mathcal{A}$ is pure by [8, Proposition 2.5 (ii)].

Theorem 4.2. The following are equivalent.

(1) $\mathcal{X}$ is absolutely pure.

(2) $\mathcal{X}$ is a pure sub-representation of an absolutely pure representation.

(3) $\mathcal{X}$ is a pure sub-representation of an injective representation.

Proof. Follows by using definitions and applying Lemma 4.1.

Lemma 4.3. Let $\mathcal{X}$ be an absolutely pure representation, Then

(i) $\mathcal{X}(v)$ is absolutely pure for each vertex $v$ of $Q$.

(ii) For any vertex $v$ of $Q$, the morphism

$$
\mathcal{X}(v) \rightarrow \mathcal{X}(t(a))
$$

is a pure epimorphism for each arrow a in $Q$.

Proof. $\mathcal{X}$ is a pure sub-representation of an injective representation $\mathcal{I}$. Thus $\mathcal{X}(v)$ is a pure submodule of an injective module $\mathcal{I}(v)$. Let $\mathcal{E}$ be the injective envelope of $\mathcal{X}, a: v \rightarrow w$ be an arrow in $Q$ and consider the following diagram.

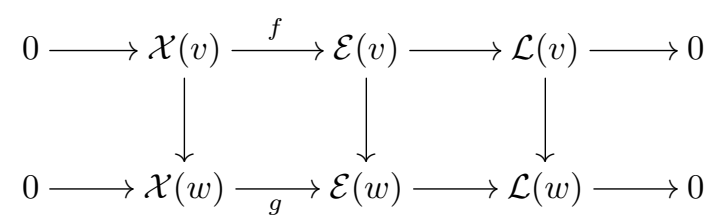


Since $\mathcal{X}$ is absolutely pure, $0 \rightarrow \mathcal{L}^{+} \rightarrow \mathcal{E}^{+} \rightarrow \mathcal{X}^{+} \rightarrow 0$ splits. Hence the diagram

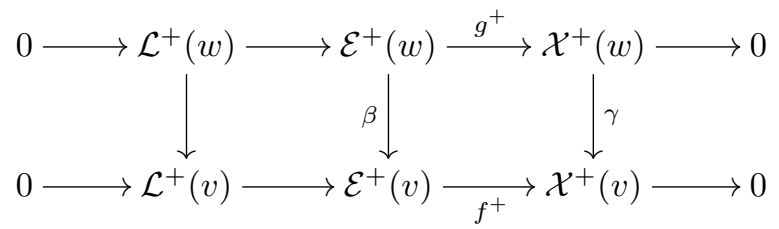

splits, i.e., there exist maps $f^{\prime}$ and $g^{\prime}$ such that $f^{+} f^{\prime}=1_{\mathcal{X}+(v)}, g^{+} g^{\prime}=1_{\mathcal{X}+(w)}$ and $\beta g^{\prime}=f^{\prime} \gamma$. Since $\beta g^{\prime}$ is monic, so is $f^{\prime} \gamma$. Hence $\gamma$ is a monomorphism. This means that $\mathcal{X}(v) \rightarrow \mathcal{X}(w)$ is an epimorphism. We can consider a homomorphism $\beta^{\prime}: \mathcal{E}^{+}(v) \rightarrow \mathcal{E}^{+}(w)$ with $\beta^{\prime} \beta=1_{\mathcal{E}^{+}(w)}$ because $\mathcal{E}$ is injective. By setting $\gamma^{\prime}:=g^{+} \beta^{\prime} f^{\prime}$ we have

$$
\gamma^{\prime} \gamma(x)=g^{+} \beta^{\prime} f^{\prime} \gamma(x)=g^{+} \beta^{\prime} \beta g^{\prime}(x)=x
$$

for each $x \in \mathcal{X}^{+}(w)$. This implies that $\mathcal{X}(v) \rightarrow \mathcal{X}(w)$ is a pure epimorphism.

Theorem 4.4. Let $Q$ be a quiver and $\mathcal{X}$ be an absolutely pure representation of $Q$. Then

(i) $\mathcal{X}(v)$ is absolutely pure for each vertex $v$ of $Q$.

(ii) For any vertex $v$ of $Q$, the morphism

$$
\mathcal{X}(v) \rightarrow \prod_{s(a)=v} \mathcal{X}(t(a))
$$

induced by $\mathcal{X}(v) \rightarrow \mathcal{X}(t(a))$ is a pure epimorphism.

Proof. By the properties of product any morphism $\mathcal{X}(v) \rightarrow \mathcal{X}(w)$ (where $a$ : $v \rightarrow w$ is an arrow in $Q$ ) factors through $\mathcal{X}(v) \rightarrow \prod_{s(a)=v} \mathcal{X}(t(a)) \rightarrow \mathcal{X}(w)$. Considering the following commutative diagram

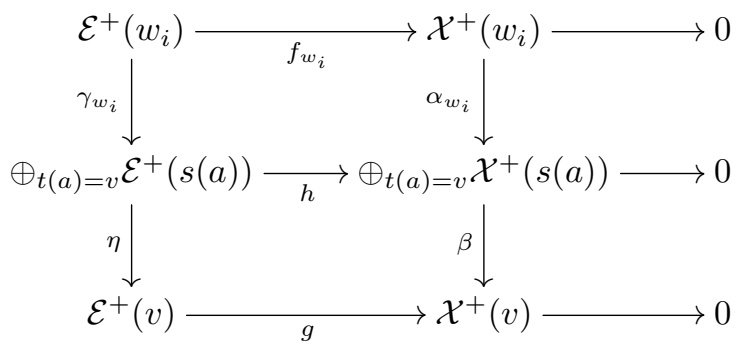

all maps except $\beta$ are split homomorphisms. Since $\mathcal{X}$ is absolutely pure, $g^{\prime} \beta \alpha_{w_{i}}=\eta \gamma_{w_{i}} f_{w_{i}}^{\prime}$. Set $\beta^{\prime}:=h \eta^{\prime} g^{\prime}$ and let $X$ be an arbitrary element in $\oplus_{w_{i}} \mathcal{X}^{+}\left(w_{i}\right)$, then $X=\sum_{i=1}^{k} \alpha_{w_{i}}\left(x_{i}\right)$ for some suitable integer $k$. We observe 
that

$$
\begin{array}{r}
\beta^{\prime} \beta(X)=h \eta^{\prime} g^{\prime} \beta\left(\sum_{i=1}^{k}\left(\alpha_{w_{i}}\left(x_{i}\right)\right)=\sum_{i=1}^{k} h \eta^{\prime} g^{\prime} \beta\left(\alpha_{w_{i}}\left(x_{i}\right)\right)\right. \\
=\sum_{i=1}^{k} h \eta^{\prime} \eta \gamma_{w_{i}} f_{w_{i}}^{\prime}\left(x_{i}\right)=\sum_{i=1}^{k} h \gamma_{w_{i}} f_{w_{i}}^{\prime}\left(x_{i}\right)=\sum_{i=1}^{k} \alpha_{w_{i}} f_{w_{i}} f_{w_{i}}^{\prime}\left(x_{i}\right)=X .
\end{array}
$$

Thus $\beta^{\prime} \beta=1_{\oplus \mathcal{X}+\left(w_{i}\right)}$ and hence $\beta$ is an split monomorphism.

Remark 4.5. A representations satisfying in the conditions of the above theorem does not need necessarily to be absolutely pure. The next example which is presented in [5, Example 3] shows that a representation of a non-right rooted quiver satisfying some conditions guaranteeing injectivity of representations over right rooted quivers may fail to be injective. It confirms our claim.

Example 2. Let us consider the non-right rooted quiver

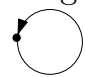

and the category of representations of $Q$ by $K$-vector spaces ( $K$ is a field). In this case $\operatorname{Rep}(Q, K)$ is equivalent to the category $K[x]$-mod. The representation $K\left[x, x^{-1}\right] \stackrel{x}{\rightarrow} K\left[x, x^{-1}\right]$ satisfies in conditions (i) and (ii) of Theorem 4.4, but $K\left[x, x^{-1}\right]$ is not injective (and hence is not absolutely pure) as a $K[x]$-module (over the Noetherian ring $K[x]$, the notions of absolute purity and injectivity are equivalent).

Definition 2.2 in [5] motivated us to consider the following definition.

Definition. A quiver $Q$ is called source absolutely pure representation quiver if for any $R$, absolutely pure representations of $\operatorname{Rep}(Q, R)$ can be characterized in terms of conditions (i) and (ii) of Theorem 4.4. We will denote the class of all source absolutely pure representation quivers by $\mathfrak{I}$.

Let $\mathfrak{Q}$ denote the class of quivers such that $Q$ does not contain $\bullet \leftarrow \bullet \rightarrow \bullet$. This is a wide class of quivers containing many important classes of quivers such as linear quivers and dual trees. By a dual tree we mean a quiver $Q$ with a vertex $v$ such that for any vertex $w$ in $Q$, there exists a unique path from $w$ to $v$. We will show that all quivers in this class are source absolutely pure.

Proposition 4.6. Let $Q$ be the quiver $v \rightarrow w$ and $\mathcal{X}$ be a representation of $Q$. $\mathcal{X}$ is absolutely pure if and only if $\mathcal{X}(v)$ and $\mathcal{X}(w)$ are both absolutely pure and $\mathcal{X}(v) \rightarrow \mathcal{X}(w)$ is a pure epimorphism.

Proof. The only if part is proved in Lemma 4.3. Conversely suppose that $\mathcal{X}$ is a representation satisfying the conditions (i) and (ii). We should show that $\mathcal{X}$ is a pure subrepresentation of an injective representation. Let $\mathcal{E}$ be the injective 
envelope of $\mathcal{X}$. Then consider the following diagram.

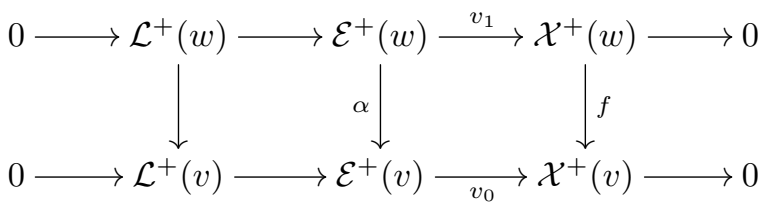

We should show that there are maps $t_{0}$ and $t_{1}$ such that $v_{1} t_{1}=1_{\mathcal{X}^{+}(w)}, v_{0} t_{0}=$ $1_{\mathcal{X}^{+}(v)}$ and $\alpha t_{1}=t_{0} f$. Since the top row splits, there is a map $t_{1}: \mathcal{X}^{+}(w) \rightarrow$ $\mathcal{E}^{+}(w)$ such that $v_{1} t_{1}=1_{\mathcal{X}^{+}(w)}$. By splitness of the second diagram, there is a map $\gamma: \operatorname{ker} g \rightarrow \mathcal{E}^{+}(v)$ such that $v_{0} \gamma=i$ where $g$ is a map satisfying $g f=$ $1_{\mathcal{X}^{+}(w)}$ and $i: \operatorname{ker} g \rightarrow \mathcal{X}^{+}(v)$ is the canonical inclusion. Set $t_{0}:=\gamma \circ \pi+\alpha t_{1} g$ where $\pi: \mathcal{X}^{+}(v) \rightarrow \operatorname{ker} g$ is the canonical projection. Then

$$
t_{0} f(x)=\gamma \circ \pi(f(x))+\alpha t_{1} g(f(x))=\alpha t_{1}(x)
$$

for each $x \in \mathcal{X}^{+}(w)$. Also we have

$$
v_{0} t_{0}(a+b)=v_{0} \gamma \pi(a+b)+v_{0} \alpha t_{1} g(a+b)=v_{0} \gamma(b)+v_{0} \alpha t_{1} g(a)=a+b
$$

where $a \in \operatorname{Im} f, b \in \operatorname{ker} g$ and $\mathcal{X}^{+}(v)=\operatorname{ker} g \oplus \operatorname{Im} f$.

The procedure can be generalized to $\mathbb{A}_{n}: \bullet \rightarrow \bullet \rightarrow \cdots \rightarrow \bullet$ for each $n \in \mathbb{N}$. For the infinite line $\mathbb{A}_{\infty}^{-}: \cdots \rightarrow \bullet \rightarrow \bullet$ and dual tree cases by using the axiom of choice, we can apply this procedure. Hence right rooted quivers in $\mathfrak{Q}$ are all source absolutely pure.

A quiver $Q$ with a vertex $v$ is called a finite tree if it has a finite set of vertices and for each vertex $w$ in $Q$ there is a unique path from $v$ to $w$.

Proposition 4.7. Every finite tree quiver is a source absolutely pure representation quiver.

Proof. The proof is similar to the proof of Proposition 4.6.

Let us present some relations between flatness and absolute purity. These relations may help us to characterize some source absolutely pure representation quivers whenever $R$ is a coherent ring.

Theorem 4.8. Let $Q$ be a left rooted quiver. Then following are equivalent.

(1) $\mathcal{X}$ is a flat representation.

(2) $\mathcal{X}^{+}$is an injective representation.

(3) $\mathcal{X}^{+}$is an absolutely pure representation.

Proof. (1) $\Rightarrow(2) \Rightarrow(3)$ follows directly.

(3) $\Rightarrow(1)$ : Let $\mathcal{X}$ be a representation with $\mathcal{X}^{+}$absolutely pure and we are 
given an exact sequence $0 \rightarrow \mathcal{Z} \rightarrow \mathcal{Y} \rightarrow \mathcal{X} \rightarrow 0$. In the diagram

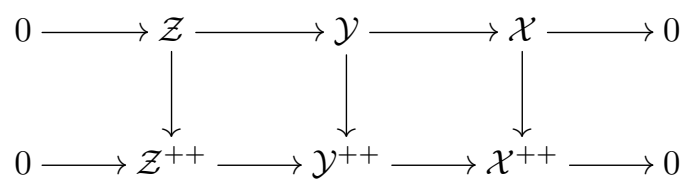

the second row splits and columns are all pure. Hence the top row is pure. So by Theorem 2.13 in [8], $\mathcal{X}$ is flat.

Proposition 4.9. Let $Q$ be a right rooted quiver and $\mathcal{X}$ be absolutely pure. Then the following are equivalent.

(1) $R$ is a coherent ring.

(2) $\mathcal{X}^{+}$is flat.

(3) $\mathcal{X}^{++}$is injective.

Proof. It is an immediate corollary of a similar result in the category of $R$ modules and Proposition 4.4.

Proposition 4.10. Let $R$ be a coherent ring and $\mathcal{X}$ be a representation of $a$ right rooted quiver $Q$ satisfying the following conditions.

(i) $\mathcal{X}(v)$ is absolutely pure for each vertex $v$ of $Q$.

(ii) For any vertex $v$ of $Q$, the morphism

$$
\mathcal{X}(v) \rightarrow \prod_{s(a)=v} \mathcal{X}(t(a))
$$

induced by $\mathcal{X}(v) \rightarrow \mathcal{X}(t(a))$ is a pure epimorphism.

Then $\mathcal{X}$ is absolutely pure.

Proof. This follows by Proposition 4.8 and Proposition 4.9.

The above proposition is also true for some non-right rooted quivers. For example for the quivers

$$
\mathbb{A}_{\infty}^{+}: \bullet \rightarrow \bullet \rightarrow \bullet \rightarrow \cdots
$$

and

$$
\mathbb{A}_{\infty}^{\infty}: \cdots \rightarrow \bullet \rightarrow \bullet \rightarrow \cdots,
$$

using Proposition 4.9 and [5, Section 5] we can check that the above proposition is true. Hence over a coherent ring a representation $\mathcal{X}$ of $\mathbb{A}_{\infty}^{+}$(or $\mathbb{A}_{\infty}^{\infty}$ ) is absolutely pure if and only if $\mathcal{X}(v)$ is absolutely pure for each vertex $v \in Q$ and $\mathcal{X}(v) \rightarrow \mathcal{X}(w)$ is a pure epimorphism for each arrow $v \rightarrow w$ in $Q$.

For a representation $\mathcal{M}$ of a quiver $Q=(V, E)$ we define $|\mathcal{M}|=\left|\cup_{v \in V} \mathcal{M}(v)\right|$.

Lemma 4.11. Let $\mathcal{M}: M_{1} \stackrel{\phi}{\rightarrow} M_{2}$ be an absolutely pure representation of the quiver $Q: \bullet \rightarrow \bullet, \aleph \geq\{|R|,|V|,|E|\}$ be an infinite cardinal and $X_{1}$ and $X_{2}$ be arbitrary subsets of $M_{1}$ and $M_{2}$ respectively. Then there exists an absolutely 
pure subrepresentation $M_{1}^{\prime} \stackrel{\phi^{\prime}}{\rightarrow} M_{2}^{\prime}$ of $M_{1} \rightarrow M_{2}$ such that $\left|M_{1}^{\prime}\right| \leq \aleph$ and $\left|M_{2}^{\prime}\right| \leq$ $\aleph$.

Proof. Let $X_{1}^{\prime}:=X_{1} \cup \phi^{-1}\left(X_{2}\right)$ and consider $\mathcal{M}^{\prime}: M_{1}^{\prime} \rightarrow M_{2}^{\prime}$ where $M_{1}^{\prime}$ is a pure submodule of $M_{1}$ containing $X_{1}^{\prime}$ with $\left|M_{1}^{\prime}\right| \leq \aleph, M_{2}^{\prime}=\phi\left(M_{1}^{\prime}\right)$ and $\phi^{\prime}=\left.\phi\right|_{M_{1}^{\prime}}$. Then $\mathcal{M}^{\prime}$ has the expected properties.

Proposition 4.12. Let $Q=(V, E)$ be a quiver in $\mathfrak{Q}$ (this may contains $\mathbb{A}_{\infty}^{+}$ and $\left.\mathbb{A}_{\infty}^{\infty}\right)$ and $\mathcal{M}$ an absolutely pure representation. Consider $\aleph$ as an infinite cardinal such that $\aleph \geq\{|R|,|V|,|E|\}$ and let $X_{v} \subseteq \mathcal{M}(v)$ be subsets with $\left|X_{v}\right| \leq$ $\aleph$ for all $v$. Then there exists an absolutely pure subrepresentation $\mathcal{M}^{\prime} \subseteq \overline{\mathcal{M}}$ with $\left|\mathcal{M}^{\prime}\right| \leq \aleph$.

Proof. The proof is similar to the proof of Proposition 3.3 in [4].

Theorem 4.13. Let $R$ be a coherent ring and $Q=(V, E)$ be a quiver in $\mathfrak{Q}$. Then the class of all absolutely pure representations of $Q$, i.e., $\operatorname{Abs}(Q)$, is a covering class.

Proof. We already have proved that $\operatorname{Cabs}(Q)$ is a covering class. By using the same way we can show that $\operatorname{Abs}(Q)$ is also a covering class.

\section{Example 3.}

(1) Let $Q: \bullet \rightarrow \bullet$ be a quiver and $\mathcal{M}: M \rightarrow 0$ be a representation where $M$ is an $R$-module with $R$ a coherent ring. Then there is an absolutely pure cover $F \rightarrow M$ for $M$. Furthermore $F \rightarrow 0$ is an absolutely pure representation since its two terms are absolutely pure and its arrow is a pure epimorphism. It is not hard to see that $(F \rightarrow 0) \longrightarrow(M \rightarrow 0)$ is an absolutely pure cover for $M \rightarrow 0$.

(2) Let $M$ be an $R$-module and $F \rightarrow M$ be its absolutely pure cover. Then for a representation $M \stackrel{i d}{\rightarrow} M$ of $\bullet \rightarrow \bullet, F \stackrel{i d}{\rightarrow} F$ is an absolutely pure cover.

\section{References}

[1] D. D. Adams, Absolutely pure modules, Ph.D. Thesis, University of Kentucky, Department of Mathematics, 1978.

[2] E. Enochs, Shortening filtrations, Sci. China Math. 55 (2012), no. 4, 687-693.

[3] E. Enochs and S. Estrada, Projective representations of quivers, Comm. Algebra 33 (2005), no. 10, 3467-3478.

4] Relative homological algebra in the category of quasi-coherent sheaves, Adv. Math. 194 (2005), no. 2, 284-295.

[5] E. Enochs, S. Estrada, and G. Rozas, Injective representations of infinite quivers. applications, Canad. J. Math. 61 (2009), no. 2, 315-335.

[6] E. Enochs, L. Oyonarte, and B. Torrecillas, Flat covers and flat representations of quivers, Comm. Algebra 32 (2004), no. 4, 1319-1338.

[7] S. Estrada and S. Özdemir, Relative homological algebra in categories of representation of quivers, Houston J. Math. 39 (2013), no. 2, 343-362. 
[8] E. Hosseini, Pure injective representations of quivers, Bull. Korean Math. Soc. 50 (2013), no. 2, 389-398.

[9] B. H. Maddox, Absolutely pure modules, Proc. Amer. Math. Soc. 18 (1967), no. 1, 155-158.

[10] C. Megibben, Absolutely pure modules, Proc. Amer. Math. Soc. 26 (1970), no. 4, 561566.

[11] S. Park, Injective and projective properties of representations of quivers with $n$ edges, Korean J Math. 16 (2008), no. 3, 323-334.

[12] K. Pinzon, Absolutely pure covers, Comm. Algebra 36 (2008), no. 6, 2186-2194.

[13] B. Stenström, Coherent rings and Fp-injective modules, J. London Math. Soc. 2 (1970), $323-329$.

MANSOUR AgHASI

Department of Mathematical SCIENCES

ISFAHAN UNIVERSITY OF TECHNOLOGY

ISFAHAN 84156-83111, IRAN

E-mail address: m.aghasi@cc.iut.ac.ir

HAmidreza Nemati

Department of Mathematical SCIEnCES

ISFAHAN UNIVERSITY OF TECHNOLOGY

ISFAHAN 84156-83111, IRAN

E-mail address: hr.nemati@math.iut.ac.ir 